Carolina Valenzuela

\section{Plantas transformables La vivienda colectiva como objeto de intervención ${ }^{1}$}

La planta puede entenderse como el material genético del espacio: y aunque no es capaz de señalar qué actos ocurrirán en él, es precisa indicando lo que nunca podrá pasar. En la vivienda colectiva, el problema que una planta tipo no puede solucionar aparece nítido. Nuevas preguntas a las tipologías, la construcción y las estrategias inmobiliarias surgen de la buisqueda de plantas capaces de cambiar, empujada por la aparición de esquemas familiares impredecibles y la atenuación de patrones sociales dominantes. Palabras clave: Vivienda colectiva, espacios flexibles, planta libre, diversidad, variabilidad, flexibilidad.

The ground plan may be understood as the genetic material of space, and though it is not able to specify which acts will occur in it, it is very precise in indicating which ones cannot. In collective housing, the problem that cannot be solved by a standard floor plan is obvious. New questions about typologies, construction and building strategies are raised by the search for floor plans that are capable of change, driven by the rise of unpredictable new family arrangements and the waning of traditionally dominant social patterns. Key words: Collective housing, flexible spaces, open plan, diversity, variability,
1 Este ensayo forma parte de la tesis titulada: Hábitats transformables. habitar y flexibilidad en el proyecto de vivienda colectiva; para optar al título de Arquitecto y al grado de Magister en Arquitectura de la Pontificia Universida Católica de Chile. El tema que aquí se y principal de dicha investigación se recopilan 34 casos de viviendas colectivas transformables del siglo $\mathrm{XX}$. Se genera una clasificación -a partir del planta libre, planta móvil, planta de recintos neutros, planta equilibrio lleno vacío, crecimiento en planta y crecimiento en corte. De acuerdo a la apacidad de variabilidad interior de las tres prim las vivendas, sẽxpos:
2 Definición de la casa tomada del "Decálogo II" de Porras incluido en avanzade p. 282. 3 Definición to Del Diccionario Española, Editorial Ramón Sopena, Barcelona, 1967
"1- La casa nunca más se entenderá como objeto, sino que será sujeto, a todos los efectos.

2- La casa se construye a sí misma, no se para en detalles.(..)

4- La casa no tiene acabados, cada material se trabaja hasta el agotamiento, siendo las juntas expresiones de sus límites.

5- Cada casa será cada vez más diferente a las demás, aunque pueda construirse estandarizadamente. 6- La casa puede cambiar de uso con facilidad, por lo que puede ser muchas o una sola. (...) 8- La casa es sinérgica, podrá desaparecer por completo o clavarse en el sitio de manera brutal, pero nunca pasivamente.

9- La casa se inserta en una red superior, inmaterial, que la liga más a la tierra que sus propios cimientos. Está abierta y penetrada por la red. (...)” Fernando Porras, $2001^{2}$

\section{La vivienda: hábitat en permanente} evolución

Transformar es “...hacer cambiar de forma. Transmutar, convertir una cosa en otra. Hacer mudar de porte, de conducta o de costumbres a algo o a alguien" 3 . En el caso de la vivienda transformar implica re-crear, porque se trata de un cambio permanente que tiene que ver con la adecuación de la casa a sus habitantes. La evolución del hábitat privado es una característica intrínseca a la especie humana: como primera capa de relación con el entorno, la vivienda es el medio donde el hombre posee su mayor poder de intervención. Es el escenario del encuentro entre éste y sus necesidades. Toda alteración, cambio, creación o modificación tiene que ver con la búsqueda de un lugar propio, un lugar donde ser y sentir pertenencia. La vivienda es el resumen del mundo, porque al igual que éste, es movida por la energía de los actos que ocurren en su interior. Dichos actos, propios a los habitantes, configuran espacios que también les son propiedad, espacios que son la construcción de un modo particular de habitar. El hombre transforma su hábitat a partir de aspectos estéticos, morfológicos, de significado y de gustos; se trata de un personalizar instintivo, relacionado a lo psicológico, lo social y lo cultural.

La vivienda es el lugar donde ocurre la vida familiar, como una serie de conflictos cambiantes o permanentes. La heterogeneidad de las formas de vivir y de habitar hace de cada vivienda un lugar adaptable, único e irrepetible. A partir de la segunda mitad del siglo XX, la coexistencia de diversas formas de vida y de costumbres otorga a la vivienda características inesperadas. La aparición de nuevas "formas familiares" enriquecen el espectro de habitantes posibles en ella: individuos solos, parejas, familias con hijos o sin ellos, ancianos, estudiantes, grupos genéricos o profesionales. La apropiación que hace cada tipo de usuario incorpora programas propios, tales como: el taller, la oficina, el lugar de estudio, la sala de juegos, etc. Por otra parte, existen hábitos actuales asociados a estas nuevas formas de vida, que dan a recintos como la cocina y el baño un protagonismo especial. Estos cambios en los modos de vida son sensibles a las nuevas experiencias tecnológicas e informáticas. Al igual que lo sucedido con el teléfono, la televisión o los electrodomésticos, la incorporación de internet en el hogar, ha hecho mutar la importancia de zonas de la vivienda asociados al trabajo o a los tiempos de ocio.

A mayor especialización de las formas de habitar, mayor existencia de identidades individuales $y$, por consecuencia, mayores necesidades de instrumentos de especialización e intervención sobre la vivienda.
Familia en la cocina, Italia, años cincuenta. Tomada de Monteys, Xavier y Fuertes, Pere; "Casa Collage: Un ensayo sobre la arquitectura de la casa". Editorial Gustavo Gili, Barcelona, 2001, p. 105

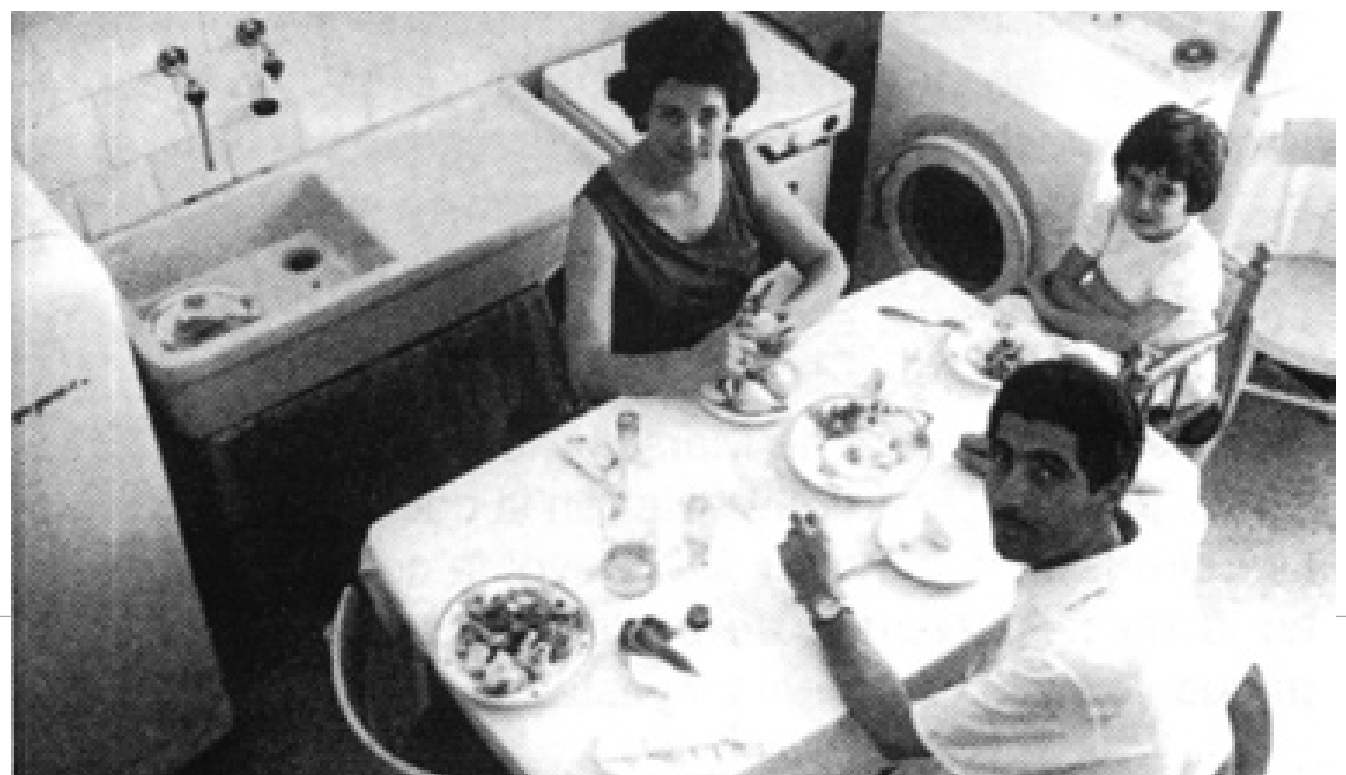


Vivienda colectiva: patrón y diferenciación La vivienda colectiva es aquella que no tiene usuario conocido. Su característica principal es que se trata de un modelo repetido un número determinado de veces en un espacio limitado. Puede ser superpuesta, pareada, o en comunidad. La dificultad de conocer al habitante futuro de cada residencia implica la adopción de la idea de usuario tipo, tomándose a la familia estándar (2 padres +2 hijos) como generadora de medidas patrón. En Chile esta vivienda de clase media se caracteriza por tener una superficie cercana a los $90 \mathrm{~m}^{2}$, distribuidos en espacios rígidos -tanto en medidas como en uso-, tales como la sala de estar, comedor, cocina, uno o dos baños y dos o tres dormitorios.

Cualquier diferencia posterior se da por intervención directa de los usuarios sobre la arquitectura cerrada. Existe una discrepancia entre la rigidez de las condiciones previas del proyecto y la variabilidad de las posteriores exigencias funcionales de los diversos ocupantes. "El método mejor, tanto desde el punto de vista pragmático como bumano, parece el de descubrir cuáles son las necesidades minimas y hacer el proyecto partiendo de abí, en forma tan libre que permita dar cabida a todas las preferencias y cambios posibles" (Rapoport, 1968).

\section{Transformabilidad interna de la planta de la vivienda colectiva}

El concepto de transformabilidad se entiende entonces como la capacidad de la vivienda colectiva de dar lugar al cambio, por medio de la incorporación a la planta de tres conceptos básicos: flexibilidad, diversidad y variabilidad. La utilización de ellos por separado o su combinación genera lo que se define como planta transformable. En el caso de la flexibilidad, se trata de proyectos que incorporan un grado de libertad que da cabida a la existencia de diversos modos de vida, basándose en la simpleza a través de la movilidad, isotropía (homogeneidad espacial) o adaptación de los elementos divisorios o componentes del interior de la vivienda. Puede existir flexibilidad física -elasticidad en la disposición espacial- o tecnológica -nuevas soluciones en la construcción y en la incorporación de nuevas tecnologías-. Cuando hay diversidad, se produce una búsqueda de variedad, desemejanza y diferenciación a través de la combinación de espacios y de elementos tecnológicos, móviles, de agrupación de programas y otros. Finalmente, el aplicar variabilidad busca no proyectar espacios definidos, sino dar lugar a la incertidumbre de la forma. A través de la dinámica de los usos cotidianos se indaga en la variación de las morfologías y recintos. Existen dos tipos de variabilidad: reversible -cambios espaciales por el ciclo de actividades diarias- e irreversible -definición espacial inicial y permanente en el tiempo-.

La planta de la vivienda colectiva puede experimentar dos tipos de mutaciones: dentro de sí misma (transformabilidad interna) o fuera de sus límites (transformabilidad externa). Ambos casos admiten cambios en la forma original, la diferencia está en que el primer grupo no altera sus límites o el cambio no tiene relación con su cáscara.

En el segundo conjunto existe una relación directa con el terreno que la acoge, es decir, puede existir crecimiento (en planta o en corte) o relaciones diversas con el entorno inmediato (espacios intermedios y llenos enfrentados a vacíos equivalentes). Para el desarrollo de la transformabilidad interna, se detallan a continuación las características de las tres tipologías definidas, ejemplificándose con casos relevantes.
I

Planta libre (pág. 76): es la ausencia de distribución o partición espacial, lo que permite un margen de libertad en cuanto a cambios de usos y jerarquías. Es una abertura del espacio ligada a la idea de liberación, porque se manifiesta a través de espacios abiertos o unitarios, supresión de pasillos, módulos regulares que definen lineamientos y estructura mínima interior. Esta tipología permite un juego de ordenaciones infinito, admitiendo la mayor variabilidad posible de la planta.

\section{II.}

Planta móvil (pág. 77): es aquella que contiene en su interior uno o más elementos móviles, que permiten la subdivisión del espacio en recintos menores y/o de tamaños diversos. Su concepto está basado en la ligereza y en la apertura a la posibilidad de diversas de plantas, proponiendo la variabilidad -reversible o irreversible- como su lema fundamental. Algunos de los elementos móviles que se incorporan son: tabiques, puertas correderas, muebles y aparatos.

\section{III.}

Planta de recintos neutros (pág. 77) : es aquella que, si bien posee habitaciones definidas e inamovibles, es capaz de proponer diversidad por la equivalencia o diferenciación en el tamaño de sus espacios. Se trata de sucesiones de recintos con una o más aberturas que permiten y definen un recorrido característico. Son espacios conectados de formas heterogéneas que admiten diferentes ordenaciones del programa requerido por sus usuarios. La intimidad se hace variable, dependiendo del modo de entender la vivienda por sus habitantes y de sus deseos de exposición o aislamiento del programa. La flexibilidad está dada en el uso de habitaciones sin nombre.
Bibliografía: AA.VV.; Mass housing. Publicaciones del Colegio Oficial de Arquitectos de Cataluña y Baleares, Barcelona, 1971. / AA, VV.; Diccionario Metápolis arquitectura avanzada. Actar, Barcelona, 2001, p. 282. / $A R Q \mathrm{~N}^{\circ} 42$ / La babitación en altura. Ediciones ARQ, Santiago, julio, 1999. / Buchanan, Peter; "Del alojamiento moderno al hogar deseado". $A V$ Monografías, $\mathrm{N}^{\circ}$ 56, Madrid, noviembre - diciembre, 1995, pp. 12 - 18. / Eleb-Vidal, Monique; Chatelet,
Anne Marie y Mandoul Thierry; "La flexibilidad como dispositivo". Quaderns, n 202, Barcelona, 1993, pp. 98 - 105. / Gausa, Manuel; Housing: Nuevas alternativas, nuevos sistemas. Editorial Actar, Barcelona, 1998. / Gausa, Manuel y Salazar, Jaime; Singular Housing: El dominio privado. Editorial Actar, Barcelona, 1999. / Gili, Gustau; Pisos piloto. Células domésticas experimentales. Editorial Gustavo Gili, Barcelona, 1997. / Hubeli, Ernst; "Lo indefinido y lo singular: La vivienda hoy o cómo dar forma a la flexibilidad". Arquitectura Viva, $\mathrm{N}^{\circ}$ 81, Barcelona, noviembre - diciembre, 2001, pp. 17 19. / Monteys, Xavier y Fuertes, Pere; Casa Collage: Un ensayo sobre la arquitectura de la casa. Editorial Gustavo Gili, Barcelona, 2001. / Rapoport, Amos; "El elemento personal en la vivienda: Una argumentación a favor del diseño abierto" (1968). En AA, VV.; Mass Housing. Publicaciones del Colegio Oficial de Arquitectos de Cataluña y Baleares, Barcelona, 1971, p. 58. 
Planta libre

1 Lofts Plaza Yungay (selección de un tipo) (Planta tercer piso, planta cuarto piso)

Arquitectos: Francisco Vergara, María José Castillo

y Margarita De Murtinho

Ubicación: Santiago, Chile 1998 - 1999

Tipo de vivienda: superpuesta

Superficie viviendas: $65 \mathrm{~m}^{2}$

Flexibilidad: física. Dos plantas libres

de divisiones interiores

Diversidad: espacios servidores fijos y

adosados al muro interior de la planta

Variabilidad: reversible. Espacios libres

de distribuciones en dos niveles

y con doble altura

2 Edificio Altamira

Arquitectos: Rafael Iglesia

Ubicación: Rosario, Argentina 1998 - 2001

Tipo de vivienda: superpuesta

Superficie viviendas: $120 \mathrm{~m}^{2}$

Flexibilidad: física. Espacio principal libre

de divisiones interiores

Diversidad: espacios servidores

Diversidad: espapados y fijos
agrupas

agrupados y fijos

libre de distribucio

Zona de espacios fijos a distinto nivel

3 Macallen Apartment Building (selección de un tipo) Arquitectos Office dA. Mónica Ponce de León y

Nader Tehrani

Ubicación: Boston, EE.UU. 2002 - 2004

Tipo de vivienda: superpuest

Flexibilidad: física. Espacio principal libre

de divisiones interiores

Diversidad: espacios servidores fijos y agrupados

en un extremo de la planta

Variabilidad: reversible. Espacio principal libre de

distribuciones y adosado al muro exterior de la planta

4 Edificio De Kaai

Arquitectos: Willem Jan Neutelings y Marc de Koonig

Ubicación: Amberes, Bélgica 1990 - 1992

Tipo de vivienda: superpuest

Superficie viviendas: $65 \mathrm{~m}^{2}$

Flexibilidad: física. Espacio central único sin divisiones

Diversidad: volumen independiente de programas

servidores fijos

Variabilidad: reversible. Distribución interior libre
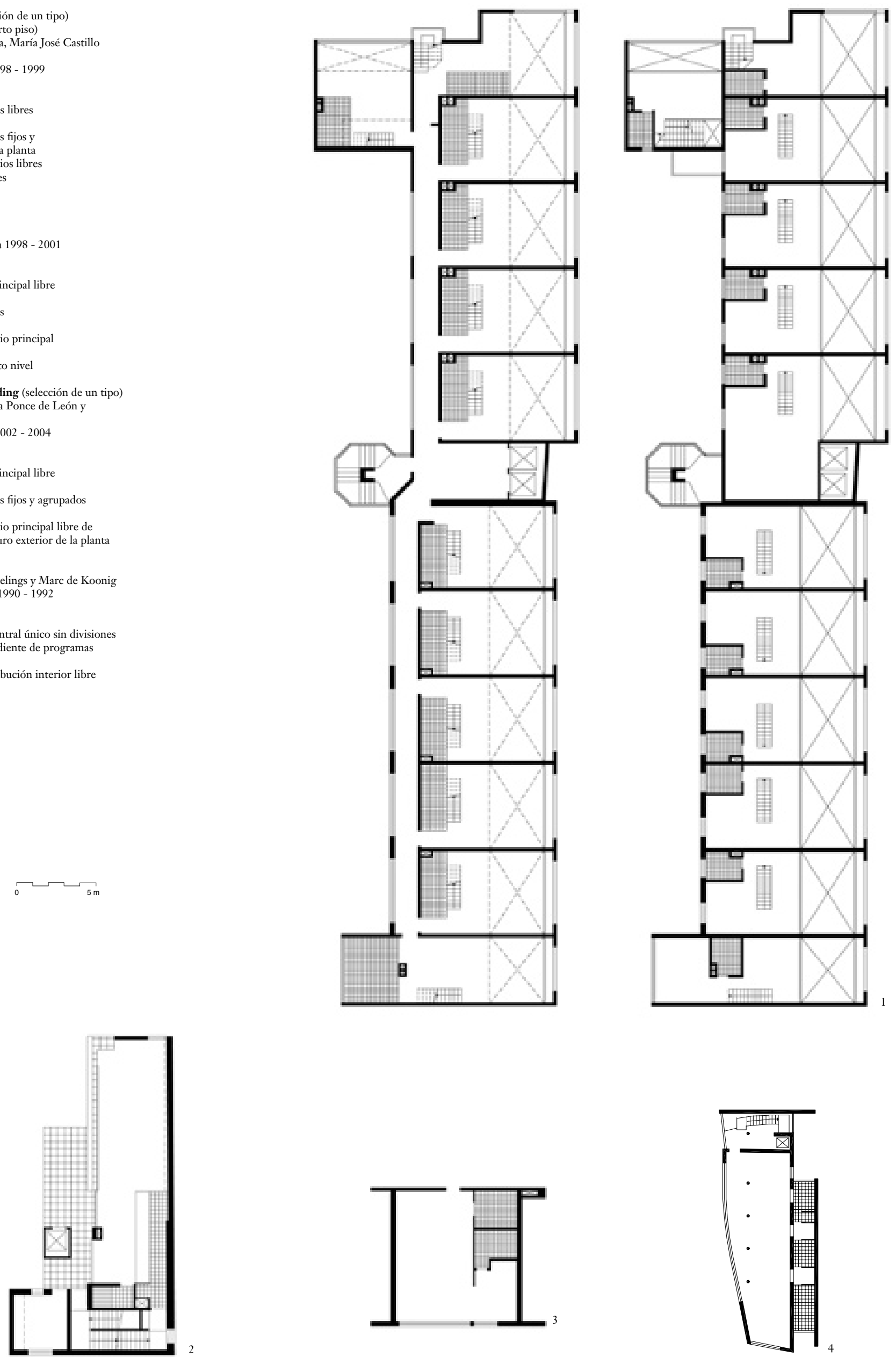
1 Viviendas para empleados de correos Arquitectos: Canale 3. Boudon, Michel, Monnot arquitectos

Ubicación: París, Francia 1989 - 1991

Tipo de vivienda: superpuesta

Superficie viviendas: $42 \mathrm{~m}^{2}$

Flexibilidad: física. Espacio único y variable

gracias a tabique giratorio

Diversidad: espacios servidores fijos y

agrupados al interior de la planta.

Posibilidad de diferenciación

programática diurna y nocturna

Variabilidad: reversible. Espacio principal

libre de distribuciones y subdivisible por la

fijación del tabique giratorio

2 Viviendas con núcleo transformable Concurso Hábitat y ciudad. (selección de un tipo)

Arquitectos: Iñaki Ábalos y Juan Herreros

Ubicación: Barcelona, España 1990

Tipo de vivienda: superpuesta

Superficie viviendas: $85 \mathrm{~m}^{2}$ y $130 \mathrm{~m}^{2}$

Flexibilidad: física y tecn

livis

divisiones y apara y tabiques móviles fijables

según una trama de $2,75 \times 2,75 \mathrm{~m}$

Diversidad: movilidad de espacios servidores

y diferenciación potencial entre programas

yer

Variabilidad: reversible. Espacio principal libre de distribucion:es y subdividible por la fijación de tabiques y aparatos móviles, generando infinitas posibilidades de plantas

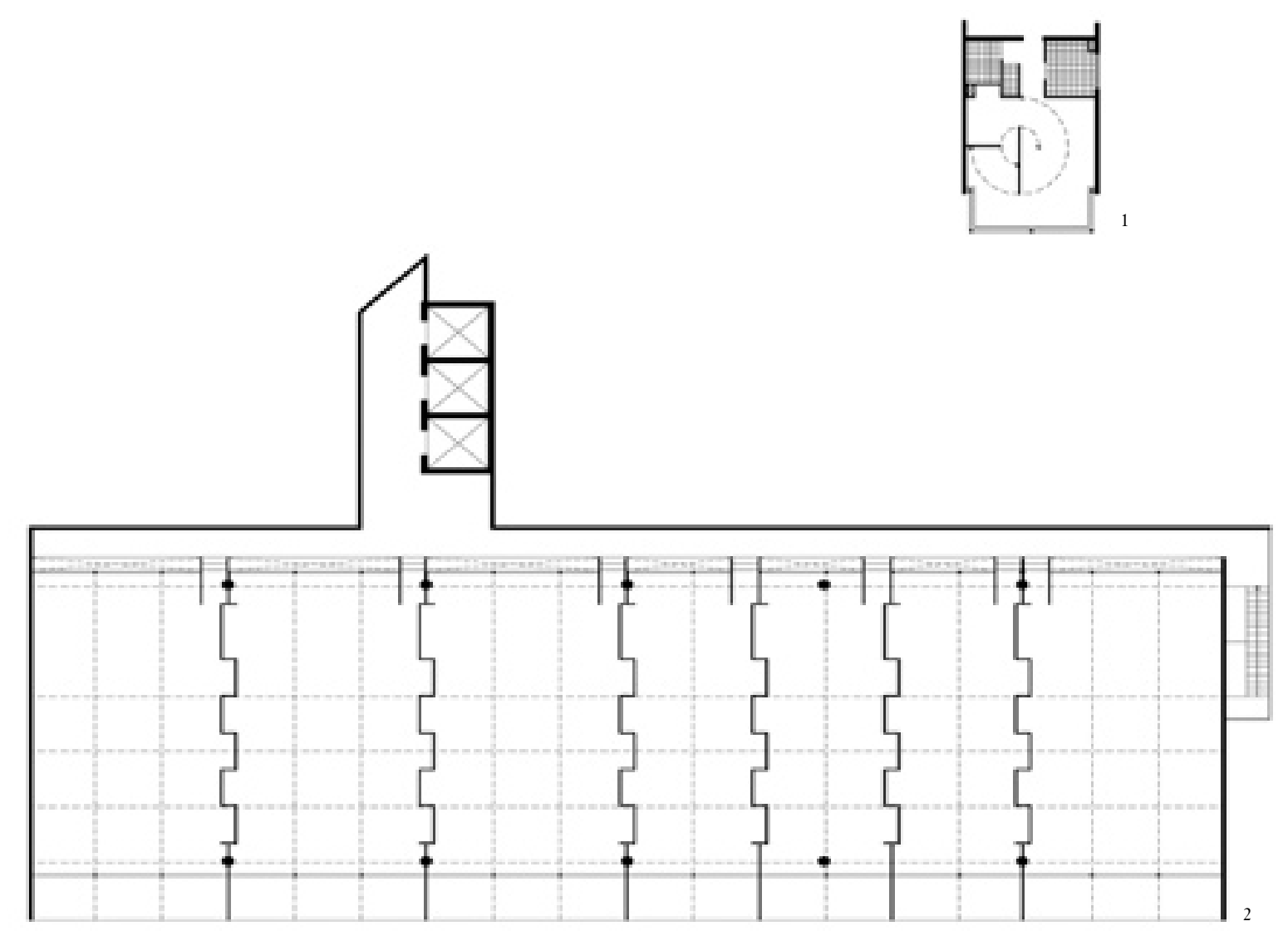

ARQ

\section{Planta de recintos neutros}

\section{Edificio Lauttasaari}

Arquitectos: Torvo Coronen y Sakari Halonen

Suomi, Finlandia 1961

Tipo de vivienda: superpuest

Superficie viviendas: $21 \mathrm{~m}^{2}, 35 \mathrm{~m}^{2}, 62 \mathrm{~m}^{2}$ y $105 \mathrm{~m}^{2}$

Flexibilidad: física. Recinto(s) relacionado(s) por

un espacio distribuidor central

Diversidad: espacios servidores fijos y agrupados

en un extremo de la planta

Variabilidad: reversible. Libertad para

concebir programas en las habitaciones interiores.

Subdivisiones fijas, pero con diversos tamaños y conexiones

2 Lofts calle Malabia

(Planta primer piso, planta segundo piso, planta tercer piso)

Arquitectos: AFRA. Armendares, Ferreiro y Rey arquitectos

Ubicación: Buenos Aires, Argentina 2000 - 2001

Tipo de vivienda: pareada

Superficie viviendas: $120 \mathrm{~m}^{2}$

Flexibilidad: física. Tres plantas superpuestas y

acceso en segundo nivel

Diversidad: espacios servidores fijos y agrupados

en un extremo de la planta

Variabilidad: reversible. Primer nivel de recintos equivalentes

(dormitorios) y seoundo y tercer nivel de espacios únicos

y doble altura. Programas distribuibles según cercanía o

lejanía al acceso y/o la calle
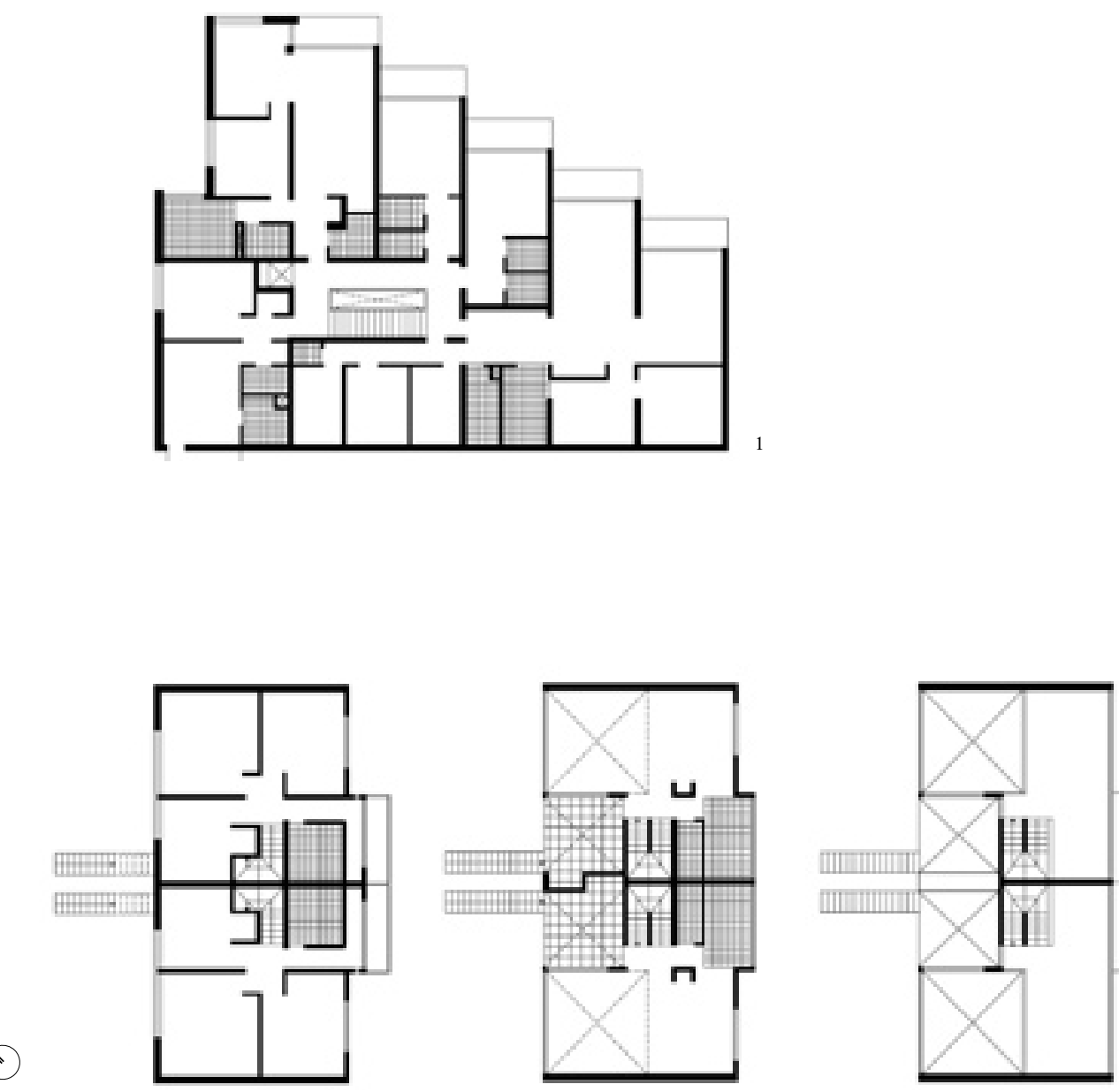

2 